\title{
Comparison of the Solution of the Van der Pol Equation Using the Modified Adomian Decomposition Method and Truncated Taylor Series Method
}

\author{
J. N. Ndam ${ }^{\mathrm{a}, *}$, O. Adedire ${ }^{\mathrm{b}}$ \\ ${ }^{a}$ Department of Mathematics, University of Jos, Nigeria \\ ${ }^{b}$ Federal College of Forestry, Jos, Nigeria
}

\begin{abstract}
In this paper, we compare the solution of the van der Pol equation obtained by using truncated Taylor series method and modified Adomian decomposition method with the solution obtained by the Poincare-Lindstedt (P-L) method. The approximating 4-component modified Adomian decomposition method behaves more like approximate P-L analytic method than tenth-order Taylor series. Also, with addition of just one term, the approximating 5-component modified Adomian decomposition method produces more convergent solution to that of P-L analytic method than the twenty second-order Taylor series approximation as the independent variable $t$ representing time progressively increases. A general comparison of the two solutions revealed that the absolute errors generated by the approximating polynomial from Taylor series are greater than the ones generated from modified Adomian decomposition method. It was further revealed that very few components of the modified Adomian decomposition could yield a series of about 3 times the order of the one obtained by using the Taylor series method. Hence, we recommend inclusion of the modified Adomian Decomposition Method in modern mathematical tools.
\end{abstract}

Keywords: van der Pol oscillator, modified Adomian decomposition method, Taylor series method

\section{Article History :}

Received: 01 January 2020

Received in revised form: 04 March 2020

Accepted for publication: 09 March 2020

Published: 14 May 2020

(C)2020 Journal of the Nigerian Society of Physical Sciences. All rights reserved. Communicated by: T. Latunde

\section{Introduction}

The van der Pol equation, otherwise called the van der Pol oscillator is a model which describes the behaviour of electrical circuits [1-19]. It was formulated by an electrical engineer and physicist, Balthasar van der Pol, and no exact solution has been obtained for the second order differential equation since then [12]. However, asymptotic and numerical solutions have been obtained. One important method of obtaining approximate analytic solutions to differential equations is the Taylor series

${ }^{*}$ Corresponding author tel. no: 07031950138

Email address: ndamj@unijos.edu.ng (J. N. Ndam) method. However, arguments have been advanced against it because the method has the problem of producing tedious computational work in determining the coefficient $a_{n}$ which can be observed in deriving recurrence relation, particularly when the product of two or more infinite series are involved [17].

Recently, another form of series solution procedure for solving both linear and nonlinear differential equations has been developed. This procedure, which generates series solutions to differential equations that converge very rapidly, is called Adomian decomposition method, named after the author [18]. The Adomian decomposition method has been used to obtain approximate analytic solutions to a wide range of differential equations, including linear and nonlinear equations, and has 
been shown to converge more rapidly with proofs of convergence than other forms of series solutions ([1, 4, 13, 17, 19]). There have been various modifications to this procedure which have significantly improved the convergence of the method ([1$3,5,8,9,14,18])$. Wazwaz [17] compared the solutions of a linear and nonlinear differential equations using the Taylor series and the Adomian decomposition methods and concluded that the solutions obtained by the decomposition method converged much more rapidly than the Taylor series procedure. However, consideration of Wazwaz [17] of the two series methods was in the sense of ease of getting Adomian decomposition series compared with that of Taylor series but did not consider error analysis of the series methods as his focus was on infinite series solution.

In this work, we attempt approximate solutions of the van der Pol equation using few orders of Taylor series and few components of the modified Adomian decomposition procedures with emphasis on finite series solution. The aim is to compare the solution of the Van der Pol equation using the Modified Adomian Decomposition Method (MADM) and the Truncated Taylor Series Method (TTSM) with approximate Poincare-Lindstedt (P-L) technique of pertubation method. The specific objectives are to investigate the behaviour of the errors generated from both methods for parameter values of van der Pol equation and to graphically display them. These objectives of the study will be achieved by comparing the solution of the van der Pol equation from finite orders of TTSM and finite components of MADM with that of approximate P-L analytic method. The choice of P-L analytic method for comparison in this study is based on its suitability and accuracy for the solution of nonlinear differential equations ([6], [11], [16]).

The remaining parts of this paper are organised as follows: the second section considers the Taylor series solution, while section 3 is dedicated to the modified Adomian decomposition procedure. Results and discussion comes up in section 4 . Finally, conclusion will be the subject of section 5 .

\section{Taylor series method}

The van der Pol oscillator is given by

$$
u^{\prime \prime}(t)+\mu\left(u^{2}(t)-1\right) u^{\prime}(t)+u(t)=0
$$

with the initial conditions

$$
u(0)=1, u^{\prime}(0)=0,
$$

where $\mu \ll 1$ or $\mu \gg 1$. The Taylor series solution about $t=0$ is obtained as

$$
u(t)=u(0)+t u^{\prime}(0)+\frac{t^{2}}{2 !} u^{\prime \prime}(0)+\frac{t^{3}}{3 !} u^{\prime \prime \prime}(0)+\cdots+\frac{t^{n}}{n !} u^{(n)}(0)(2)
$$

Equation (1) can be written as

$$
\begin{gathered}
u^{\prime \prime}(t)=\mu\left(1-u^{2}(t)\right) u^{\prime}(t)-u(t) \\
u^{\prime \prime \prime}(t)=-2 \mu u(t) u^{\prime 2}(t)+\mu\left(1-u^{2}(t)\right) u^{\prime \prime}(t)-u^{\prime}(t)
\end{gathered}
$$

$$
\begin{aligned}
u^{i v}(t)= & -2 \mu u^{\prime 3}-6 \mu u(t) u^{\prime}(t) u^{\prime \prime}(t)-8 \mu u(t) u^{\prime}(t) u^{\prime \prime \prime}(t) \\
& +\mu\left(1-u(t)^{2}\right) u^{i v}(t)-u^{\prime \prime \prime}(t)
\end{aligned}
$$

Computing the derivatives as far as possible and evaluating at $t=0$, we obtain

$$
\begin{gathered}
u(0)=1 \\
u^{\prime}(0)=0 \\
u^{(2)}(0)=-1 \\
u^{(3)}(0)=0 \\
u^{(4)}(0)=1 \\
u^{(5)}(0)=-6 \mu \\
u^{(6)}(0)=-1 \\
u^{(7)}(0)=66 \mu \\
u^{(8)}(0)=-252 \mu^{2}+1 \\
u^{(9)}(0)=-612 \mu \\
u^{(10)}(0)=11052 \mu^{2}-1 \\
u^{(11)}(0)=5532 \mu-31752 \mu^{3} \\
u^{(12)}(0)=-341316 \mu^{2}+1 \\
u^{(13)}(0)=-49818 \mu+3714552 \mu^{3} \\
u^{(14)}(0)=6259644 \mu^{2}-8845200 \mu^{4}-1 \\
u^{(15)}(0)=448398 \mu-274369248 \mu^{3} \\
u^{(16)}(0)=2211091344 \mu^{4}-241794792 \mu^{2}+1 \\
(0)-4604457312 \mu^{5}+15983461728 \mu^{3}-4035384 \mu
\end{gathered}
$$

Using the above values, tenth-order Taylor series yields the following approximating polynomial:

$$
\begin{aligned}
u(t) \approx & 1-\frac{t^{2}}{2 !}+\frac{t^{4}}{4 !}-6 \mu \frac{t^{5}}{5 !}+\frac{t^{6}}{6 !}+66 \mu \frac{t^{7}}{7 !} \\
& -\left(252 \mu^{2}+1\right) \frac{t^{8}}{8 !}-612 \mu \frac{t^{9}}{9 !}+\left(11052 \mu^{2}-1\right) \frac{t^{10}}{10 !}
\end{aligned}
$$

Analogously, twenty second-order Taylor series yields the following approximating polynomial:

$$
\begin{aligned}
& u(t) \approx 1-\frac{t^{2}}{2 !}+\frac{t^{4}}{4 !}-6 \mu \frac{t^{5}}{5 !}+\frac{t^{6}}{6 !}+66 \mu \frac{t^{7}}{7 !} \\
& -\left(252 \mu^{2}+1\right) \frac{t^{8}}{8 !}-612 \mu \frac{t^{9}}{9 !}+\left(11052 \mu^{2}-1\right) \frac{t^{10}}{10 !} \\
& +\left(5532 \mu-31752 \mu^{3}\right) \frac{t^{11}}{11 !}-\left(341316 \mu^{2}-1\right) \frac{t^{12}}{12 !} \\
& -\left(49818 \mu-3714552 \mu^{3}\right) \frac{t^{13}}{13 !}+\left(6259644 \mu^{2}-8845200 \mu^{4}\right. \\
& -1) \frac{t^{14}}{14 !}+\left(448398 \mu-274369248 \mu^{3}\right) \frac{t^{15}}{15 !}
\end{aligned}
$$




$$
\begin{aligned}
& +\left(2211091344 \mu^{4}-241794792 \mu^{2}+1\right) \frac{t^{16}}{16 !} \\
& +\left(-4604457312 \mu^{5}+15983461728 \mu^{3}-4035384 \mu\right) \frac{t^{17}}{17 !} \\
& +\left(-325537770960 \mu^{4}+-325537770960 \mu^{2}-1\right) \frac{t^{18}}{18 !} \\
& +\left(2136491544096 \mu^{5}-924215233680 \mu^{3}\right. \\
& +36320664 \mu) \frac{t^{19}}{19 !}+\left(-4018487578560 \mu^{6}\right. \\
& \left.+37614999137616 \mu^{4}-154525796280 \mu^{2}+1\right) \frac{t^{20}}{20 !} \\
& +\left(-557011268077248 \mu^{5}+48491799847920 \mu^{3}\right. \\
& -326886030 \mu) \frac{t^{21}}{21 !}+\left(3140584492618944 \mu^{6}\right. \\
& \left.-3803681636636400 \mu^{4}+3873523538520 \mu^{2}-1\right) \frac{t^{22}}{22 !}
\end{aligned}
$$

\section{Adomian decomposition method}

In this section, we follow the approach used by El-Kalla [4] in which he considered $k^{\text {th }}$ order nonlinear ordinary differential equation whose nonlinear term has Adomian polynomial representation. This approach is similar to that of Wazwaz [17]. The Adomian decomposition procedure seeks a solution of the form

$$
u(t)=\sum_{n=0}^{\infty} u_{n}(t)
$$

Equation (1) can be expressed in operator form as

$$
L_{t} u=\mu\left(1-u^{2}\right) u^{\prime}-u
$$

where $L_{t}=\frac{d^{2}}{d t^{2}}$, and hence the solution of (1) can be expressed as

$$
u(t)=u(0)+L_{t}^{-1}\left\{\mu\left(u^{\prime}-u^{2} u^{\prime}\right)-u\right\}
$$

where $L_{t}^{-1}=\iint() d t d$.$t is the two-fold integration operator.$ Initial condition of eq. (1) given by $u(0)=1$ is denoted by $u_{0}=1$ so that from eq. (5), the components that make up the series becomes

$$
\begin{aligned}
& u_{0}=1 \\
& u_{n+1}(t)=\int_{0}^{t} \int_{0}^{t}\left\{\mu\left(\left(u_{n}(s)\right)^{\prime}-A_{n}(s)\right)-u_{n}(s)\right\} d s d s
\end{aligned}
$$

where

$$
A_{n}=\frac{1}{n !} \frac{d^{n}}{d \lambda^{n}}\left[N\left(\sum_{i=0}^{\infty} u_{i} \lambda^{i}\right)\right]_{\lambda=0}=u_{n}^{2}\left(u_{n}\right)^{\prime}
$$

$n=0,1,2, \cdots$ and $\lambda$ is a parameter, are called the Adomian polynomials. However, we shall use the modified procedure for generating the Adomian polynomials as discussed below:

Suppose the nonlinear term in (1) is denoted by $N(u)$, then we decompose it as

$$
A_{0}=N\left(u_{0}\right)
$$

$$
\begin{gathered}
A_{1}=N\left(u_{0}+u_{1}\right)-A_{0} \\
A_{2}=N\left(u_{0}+u_{1}+u_{2}\right)-\left(A_{0}+A_{1}\right) \\
A_{3}=N\left(u_{0}+u_{1}+u_{2}+u_{3}\right)-\left(A_{0}+A_{1}+A_{2}\right)
\end{gathered}
$$

Thus we obtain the relation for generating the Adomian polynomials as

$$
A_{n}=N\left(\sum_{i=0}^{n} u_{i}\right)-\sum_{i=0}^{n-1} A_{i}
$$

From the initial value problem (1), $N(u)=u^{2} u^{\prime}$ and hence by using (10), the Adomian polynomials are obtained as

$$
\begin{gathered}
A_{0}=u_{0}^{2} u_{0}^{\prime} \\
A_{1}=\left(u_{0}+u_{1}\right)^{2}\left(u_{0}^{\prime}+u_{1}^{\prime}\right)-u_{0}^{2} u_{0}^{\prime} \\
=u_{0}^{2} u_{1}^{\prime}+2 u_{0} u_{1} u_{0}^{\prime}+2 u_{0} u_{1} u_{1}^{\prime}+u_{1}^{2} u_{0}^{\prime}+u_{1}^{2} u_{1}^{\prime} \\
A_{2}=\left(u_{0}+u_{1}+u_{2}\right)^{2}\left(u_{0}^{\prime}+u_{1}^{\prime}+u_{2}^{\prime}\right)-\left(A_{0}+A_{1}\right) \\
=u_{0}^{2} u_{2}^{\prime}+2 u_{0} u_{1} u_{2}^{\prime}+2 u_{0} u_{2} u_{0}^{\prime}+2 u_{0} u_{2} u_{1}^{\prime} \\
+2 u_{0} u_{2} u_{2}^{\prime}+2 u_{1} u_{2} u_{0}^{\prime}+2 u_{1} u_{2} u_{1}^{\prime}+2 u_{1} u_{2} u_{2}^{\prime} \\
+u_{2}^{2} u_{1}^{\prime}+u_{2}^{2} u_{2}^{\prime}+u_{1}^{2} u_{2}^{\prime}+u_{2}^{2} u_{0}^{\prime}
\end{gathered}
$$

Accordingly, we obtain

$$
u_{0}=1
$$

$$
u_{1}=-\frac{t^{2}}{2}
$$

$$
u_{2}=\frac{\mu}{168} t^{7}-\frac{\mu}{20} t^{5}+\frac{t^{4}}{4 !}
$$

$u_{3}=-\frac{\mu^{4}}{312947712} t^{22}+\frac{\mu^{4}}{11289600} t^{20}-\frac{\mu^{4}}{1299600} t^{18}-\frac{\mu^{3}}{12870144} t^{19}$

$+\frac{\mu^{3}}{399840} t^{17}-\frac{\mu}{240}\left(\frac{5 \mu}{32256}-\frac{\mu^{3}}{1600}\right) t^{16}-\frac{617 \mu^{3}}{21168000} t^{15}+\frac{3 \mu^{2}}{125440} t^{14}$

$-\frac{\mu}{156}\left(-\frac{31 \mu^{2}}{1400}+\frac{1}{3456}\right) t^{13}-\frac{41 \mu^{2}}{120960} t^{12}-\frac{\mu}{110}\left(-\frac{5}{576}+\frac{\mu^{2}}{40}\right) t^{11}$

$$
+\frac{19 \mu^{2}}{8400} t^{10}-\frac{13 \mu}{9072} t^{9}-\frac{\mu^{2}}{160} t^{8}+\frac{\mu}{140} t^{7}-\frac{t^{6}}{6 !}
$$

The series solution for 4-components Adomian decomposition then becomes

$$
u(t)=u_{0}+u_{1}+u_{2}+u_{3}
$$

which gives

$$
\begin{aligned}
& u(t)=1-\frac{t^{2}}{2}+\frac{\mu}{168} t^{7}-\frac{\mu}{20} t^{5}+\frac{t^{4}}{4 !} \\
& -\frac{\mu^{4}}{312947712} t^{22}+\frac{\mu^{4}}{11289600} t^{20}
\end{aligned}
$$




$$
\begin{aligned}
& -\frac{\mu^{4}}{1299600} t^{18}-\frac{\mu^{3}}{12870144} t^{19}+\frac{\mu^{3}}{399840} t^{17} \\
& -\frac{\mu}{240}\left(\frac{5 \mu}{32256}-\frac{\mu^{3}}{1600}\right) t^{16} \\
& -\frac{617 \mu^{3}}{21168000} t^{15}+\frac{3 \mu^{2}}{125440} t^{14} \\
& -\frac{\mu}{156}\left(-\frac{31 \mu^{2}}{1400}+\frac{1}{3456}\right) t^{13} \\
& -\frac{41 \mu^{2}}{120960} t^{12}-\frac{\mu}{110}\left(-\frac{5}{576}\right. \\
& \left.+\frac{\mu^{2}}{40}\right) t^{11}+\frac{19 \mu^{2}}{8400} t^{10} \\
& -\frac{13 \mu}{9072} t^{9}-\frac{\mu^{2}}{160} t^{8}+\frac{\mu}{140} t^{7}-\frac{t^{6}}{6 !}
\end{aligned}
$$

and the series solution for 5-component Adomian decomposition then gives

$$
u(t)=u_{0}+u_{1}+u_{2}+u_{3}+u_{4}
$$

We simplify (13) and choose $\mu$ to be arbitrarily small because approximate P-L method to be used for comparison can work effectively on differential equations of the form

$$
u^{\prime \prime}+w_{0}^{2} u=\mu F\left(t, u, u^{\prime}\right), 0<\mu<<1
$$

whose leading order is oscillatory with frequency $w_{0}[10]$. So eq.(13) using $\mu=2 \times 10^{-2}$ gives

$$
\begin{aligned}
& u(t)=1-1 / 2 t^{2}+0.0002619047619 t^{7} \\
& -0.001000000000 t^{5}-\frac{t^{6}}{720}+1 / 24 t^{4} \\
& +1.329776164 \times 10^{-50} t^{67}-1.139867761 \times 10^{-48} t^{65} \\
& +5.077535944 \times 10^{-47} t^{64}+4.357672234 \times 10^{-47} t^{63} \\
& -4.592882729 \times 10^{-45} t^{62}+8.593423967 \times 10^{-44} t^{61} \\
& -8.269818866 \times 10^{-42} t^{59}-1.845062816 \times 10^{-13} t^{23} \\
& +1.866090683 \times 10^{-43} t^{60}+8.309132396 \times 10^{-41} t^{58} \\
& +3.568516858 \times 10^{-40} t^{57}-8.714821000 \times 10^{-39} t^{56} \\
& +4.811105327 \times 10^{-38} t^{55}+4.002845402 \times 10^{-37} t^{54} \\
& -5.886109478 \times 10^{-36} t^{53}+1.424302351 \times 10^{-35} t^{52} \\
& +2.902430667 \times 10^{-34} t^{51}-2.595201219 \times 10^{-33} t^{50} \\
& -1.043753849 \times 10^{-33} t^{49}+1.407479522 \times 10^{-31} t^{48} \\
& -7.032084376 \times 10^{-31} t^{47}-3.022579830 \times 10^{-30} t^{46} \\
& +4.519343938 \times 10^{-29} t^{45}-8.093847241 \times 10^{-29} t^{44} \\
& -1.418636597 \times 10^{-27} t^{43}+8.881156719 \times 10^{-27} t^{42} \\
& +1.617446587 \times 10^{-26} t^{41}-3.583414553 \times 10^{-25} t^{40} \\
& +7.270414170 \times 10^{-25} t^{39}+8.689959175 \times 10^{-24} t^{38} \\
& -4.931206414 \times 10^{-23} t^{37}-1.020281668 \times 10^{-22} t^{36} \\
& +1.652954579 \times 10^{-21} t^{35}-1.931801068 \times 10^{-21} t^{34} \\
& -3.707212443 \times 10^{-20} t^{33}+1.482428707 \times 10^{-19} t^{32} \\
& +5.109844495 \times 10^{-19} t^{31}-4.724699345 \times 10^{-18} t^{30}
\end{aligned}
$$

$+2.377613320 \times 10^{-19} t^{29}+1.016506228 \times 10^{-16} t^{28}$

$-2.556365546 \times 10^{-16} t^{27}-1.543943729 \times 10^{-15} t^{26}$ $+8.696083800 \times 10^{-15} t^{25}+1.257108256 \times 10^{-14} t^{24}$ $+1.611259824 \times 10^{-13} t^{22}-9.420685884 \times 10^{-12} t^{20}$ $+0.00002230158730 t^{8}+2.799612144 \times 10^{-12} t^{21}$

$-3.001809900 \times 10^{-11} t^{19}+0.0000000002238474420 t^{18}$

$+0.0000000001499617141 t^{17}-0.000000003494172630 t^{16}$ $+0.000000003368584091 t^{15}+0.00000003773049937 t^{14}$ $-0.0000001349519000 t^{13}-0.0000002741969964 t^{12}$ $+0.000002674211961 t^{11}+0.000001218253968 t^{10}$ $-0.00003373015873 t^{9}$

\section{Results and Discussion}

Here, comparison of results from the approximating polynomials (3), (4), (12) and (14) with approximate P-L method for the solution of (1) and its initial conditions are shown in Tables 1, 2, 3 and 4. The choice of approximate P-L method is because exact solution has not been obtained for the Van der Pol equation. Details of the approximate method used here can be obtained from ([10], [15]) and references contained therein. It should be noted that the initial conditions used in this research suggest that the periodic behaviour exhibits the amplitude 1 as against the amplitude 2 used in [15]. Absolute errors (ABS Errors) are also obtained and are tabulated as follows:

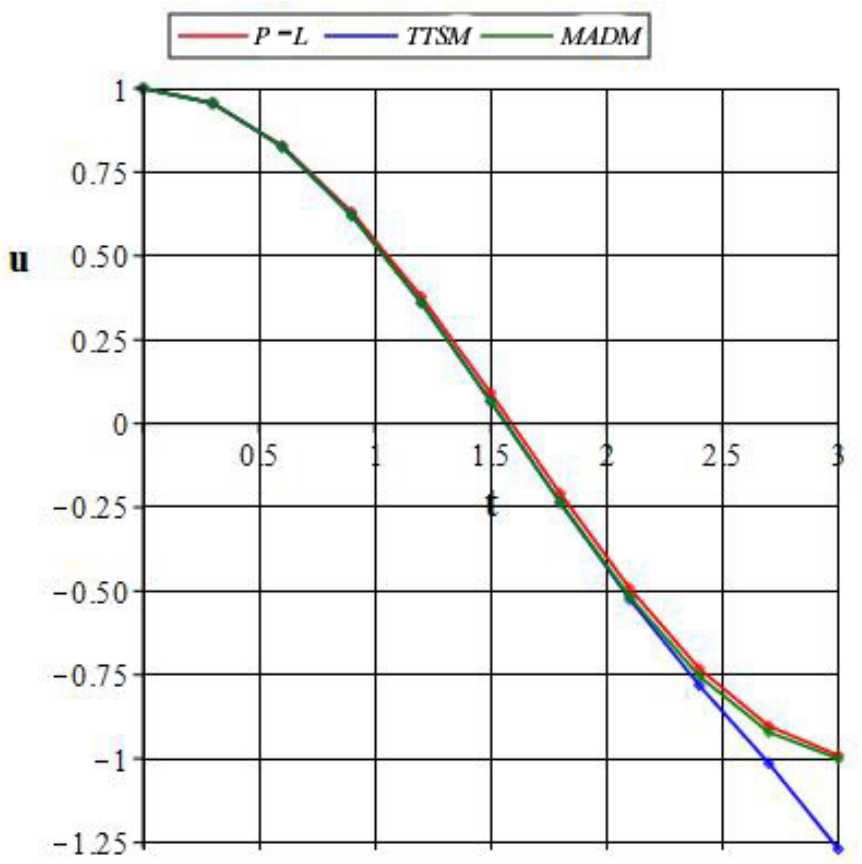

Figure 1: Graphical solutions using P-L method and approximating polynomials (3) and (12) of TTSM and 4-component MADM.

While the graphs of solutions of equation (1) and its initial conditions using approximate P-L analytic method and approximating polynomials (3), (4), (12) and (14) are shown in Figures 
Table 1: Solutions and errors from (3) and (12) of TTSM series and 4-component MADM

\begin{tabular}{lccccr}
\hline $\mathrm{t}$ & Poincare-Lindstedt & TTSM & MADM & ABS Error TTSM & ABS Error MADM \\
\hline 0 & 1.000000000000 & 1.000000000000 & 1.000000000000 & 0.000000000000 & 0.000000000000 \\
0.3 & 0.955854836508 & 0.955334115584 & 0.955334114058 & 0.000520720924 & 0.000520722450 \\
0.6 & 0.828944246584 & 0.825264812016 & 0.825264451699 & 0.003679434568 & 0.003679794885 \\
0.9 & 0.631240082296 & 0.621131026775 & 0.621122751091 & 0.010109055521 & 0.010117331205 \\
1.2 & 0.378578337374 & 0.360630621478 & 0.360560141843 & 0.017947715896 & 0.018018195531 \\
1.5 & 0.0906245246636 & 0.066327550293 & 0.065998964725 & 0.024296974371 & 0.024625559939 \\
1.8 & -0.20868615269 & -0.23659702897 & -0.23752492072 & 0.027910876280 & 0.028838768030 \\
2.1 & -0.491935529476 & -0.52423546969 & -0.52542008925 & 0.032299940214 & 0.033484559774 \\
2.4 & -0.731188366817 & -0.78110829991 & -0.77773900461 & 0.049919933093 & 0.046550637793 \\
2.7 & -0.902481373797 & -1.01309133371 & -0.98448885633 & 0.110609959913 & 0.082007482533 \\
3.0 & -0.989925613354 & -1.26963992857 & -1.15176986217 & 0.279714315216 & 0.161844248816 \\
\hline
\end{tabular}

Table 2: Solutions and errors from (4) and (12) of TTSM and 4-component MADM

\begin{tabular}{lccccr}
\hline $\mathrm{t}$ & Poincare-Lindstedt & TTSM & MADM & ABS Error TTSM & ABS Error MADM \\
\hline 0 & 1.000000000000 & 1.000000000000 & 1.000000000000 & 0.000000000000 & 0.000000000000 \\
0.3 & 0.955854836508 & 0.955334115588 & 0.955334114058 & 0.000520720920 & 0.000520722450 \\
0.6 & 0.828944246584 & 0.825264821264 & 0.825264451699 & 0.003679425320 & 0.003679794885 \\
0.9 & 0.631240082296 & 0.621131785279 & 0.621122751091 & 0.010108297017 & 0.010117331205 \\
1.2 & 0.378578337374 & 0.360647539325 & 0.360560141843 & 0.017930798049 & 0.018018195531 \\
1.5 & 0.0906245246636 & 0.066512092157 & 0.065998964725 & 0.024112432507 & 0.024625559939 \\
1.8 & -0.20868615269 & -0.23531787096 & -0.23752492072 & 0.026631718270 & 0.028838768030 \\
2.1 & -0.491935529476 & -0.51774914669 & -0.52542008925 & 0.025813617214 & 0.033484559774 \\
2.4 & -0.731188366817 & -0.75481924354 & -0.77773900461 & 0.023630876723 & 0.046550637793 \\
2.7 & -0.902481373797 & -0.9216069535 & -0.98448885633 & 0.019125579703 & 0.082007482533 \\
3.0 & -0.989925613354 & -0.97208752941 & -1.15176986217 & 0.017838083944 & 0.161844248816 \\
\hline
\end{tabular}

Table 3: Solutions and errors from (3) and (14) of TTSM and 5-component MADM

\begin{tabular}{lccccr}
\hline $\mathrm{t}$ & Poincare-Lindstedt & TTSM & MADM & ABS Error TTSM & ABS Error MADM \\
\hline 0 & 1.000000000000 & 1.000000000000 & 1.000000000000 & 0.000000000000 & 0.000000000000 \\
0.3 & 0.955854836508 & 0.955334115584 & 0.95533411559 & 0.000520720924 & 0.000520720918 \\
0.6 & 0.828944246584 & 0.825264812016 & 0.825264822641 & 0.003679434568 & 0.003679423943 \\
0.9 & 0.631240082296 & 0.621131026775 & 0.621131859045 & 0.010109055521 & 0.010108223251 \\
1.2 & 0.378578337374 & 0.360630621478 & 0.360648788531 & 0.017947715896 & 0.017929548843 \\
1.5 & 0.0906245246636 & 0.066327550293 & 0.066523502482 & 0.024296974371 & 0.024101022182 \\
1.8 & -0.20868615269 & -0.23659702897 & -0.23524709918 & 0.027910876280 & 0.026560946490 \\
2.1 & -0.491935529476 & -0.52423546969 & -0.51742153967 & 0.032299940214 & 0.025486010194 \\
2.4 & -0.731188366817 & -0.78110829991 & -0.75375297048 & 0.049919933093 & 0.022564603663 \\
2.7 & -0.902481373797 & -1.01309133371 & -0.92099274209 & 0.110609959913 & 0.018511368293 \\
3.0 & -0.989925613354 & -1.26963992857 & -0.99998289494 & 0.279714315216 & 0.010057281586 \\
\hline
\end{tabular}


J. N. Ndam E O. Adedire/J. Nig. Soc. Phys. Sci. 2 (2020) 106-114

Table 4: Solutions and errors from (4) and (14) of TTSM and 5-component MADM

\begin{tabular}{lccccr}
\hline $\mathrm{t}$ & Poincare-Lindstedt & TTSM & MADM & ABS Error TTSM & ABS Error MADM \\
\hline 0 & 1.000000000000 & 1.000000000000 & 1.000000000000 & 0.000000000000 & 0.000000000000 \\
0.3 & 0.955854836508 & 0.955334115588 & 0.95533411559 & 0.000520720920 & 0.000520720918 \\
0.6 & 0.828944246584 & 0.825264821264 & 0.825264822641 & 0.003679425320 & 0.003679423943 \\
0.9 & 0.631240082296 & 0.621131785279 & 0.621131859045 & 0.010108297017 & 0.010108223251 \\
1.2 & 0.378578337374 & 0.360647539325 & 0.360648788531 & 0.017930798049 & 0.017929548843 \\
1.5 & 0.0906245246636 & 0.066512092157 & 0.066523502482 & 0.024112432507 & 0.024101022182 \\
1.8 & -0.20868615269 & -0.23531787096 & -0.23524709918 & 0.026631718270 & 0.026560946490 \\
2.1 & -0.491935529476 & -0.51774914669 & -0.51742153967 & 0.025813617214 & 0.025486010194 \\
2.4 & -0.731188366817 & -0.75481924354 & -0.75375297048 & 0.023630876723 & 0.022564603663 \\
2.7 & -0.902481373797 & -0.9216069535 & -0.92099274209 & 0.019125579703 & 0.018511368293 \\
3.0 & -0.989925613354 & -0.97208752941 & -0.99998289494 & 0.017838083944 & 0.010057281586 \\
\hline
\end{tabular}

1, 2, 3 and 4 for $\mu=2 \times 10^{-2}$, the graphs of absolute errors are also indicated in Figures 5, 6, 7 and 8.

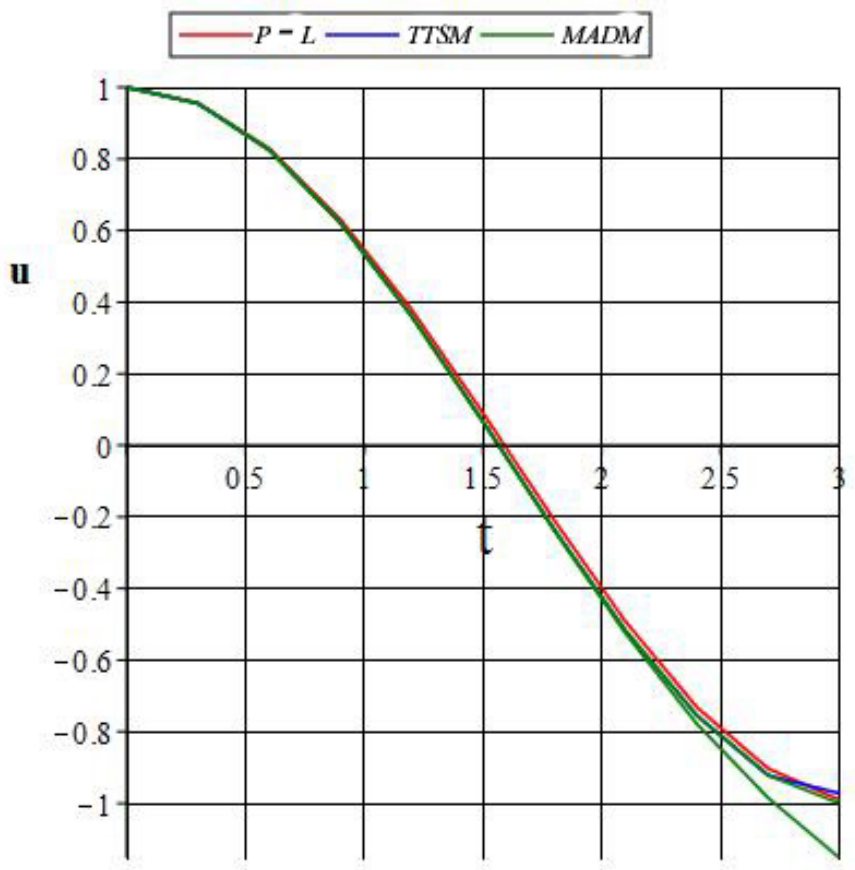

Figure 2: Graphical solutions using P-L method and approximating polynomials (4) and (12) of TTSM and 4-component MADM.

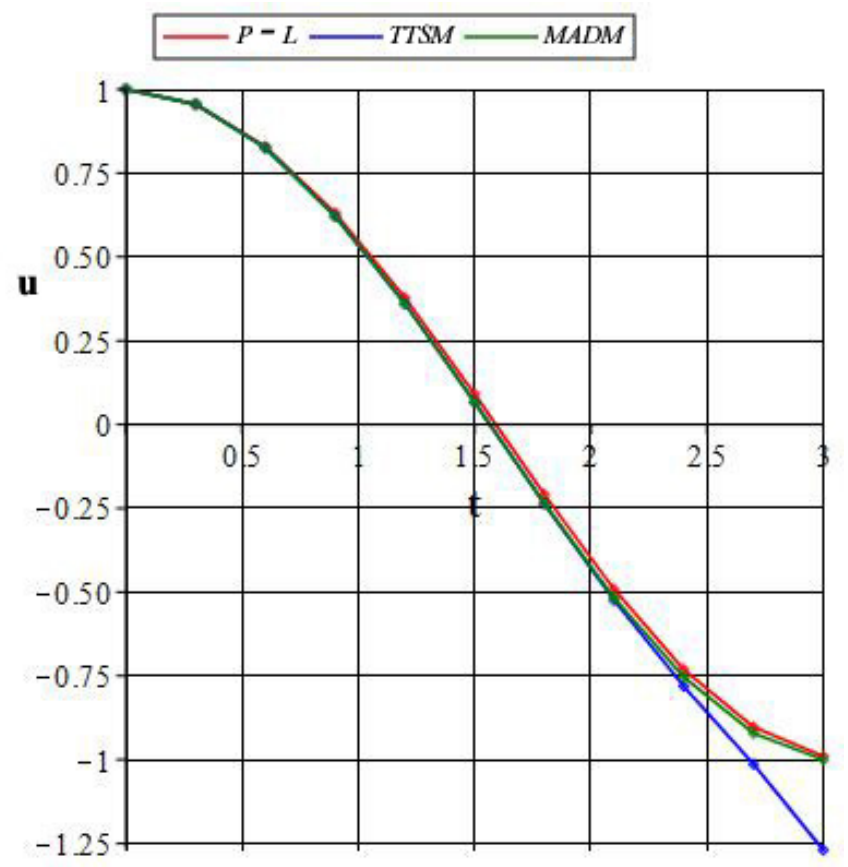

Figure 3: Graphical solutions using P-L method and approximating polynomials (3) and (14) of TTSM and 5-component MADM. 


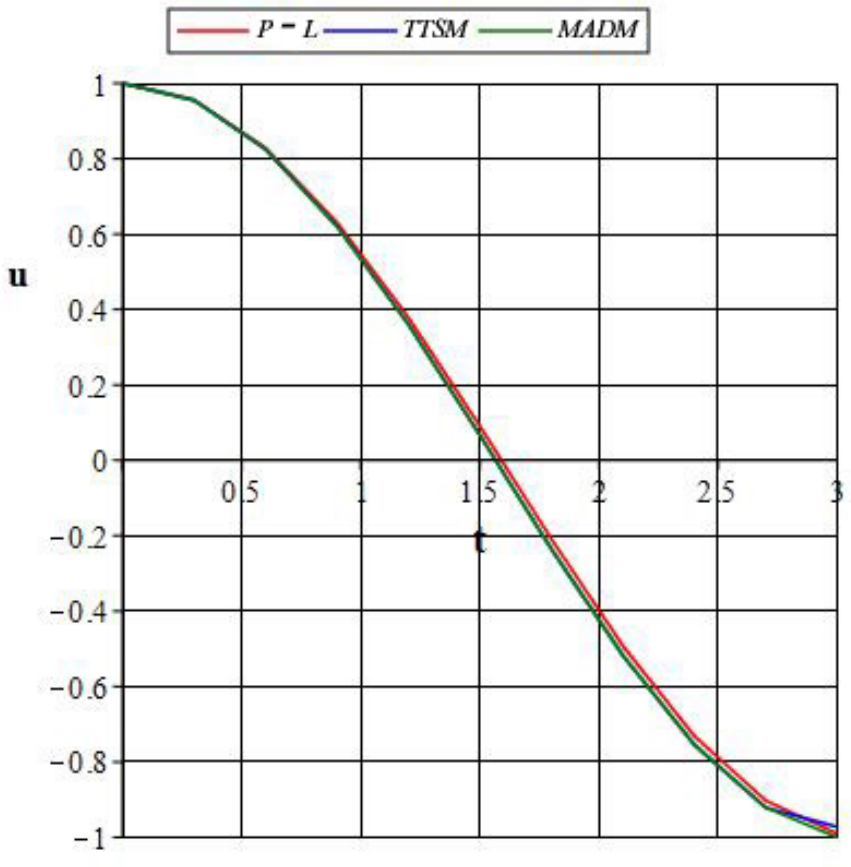

Figure 4: Graphical solutions using P-L method and approximating polynomials (4) and (14) of TTSM and 5-component MADM.

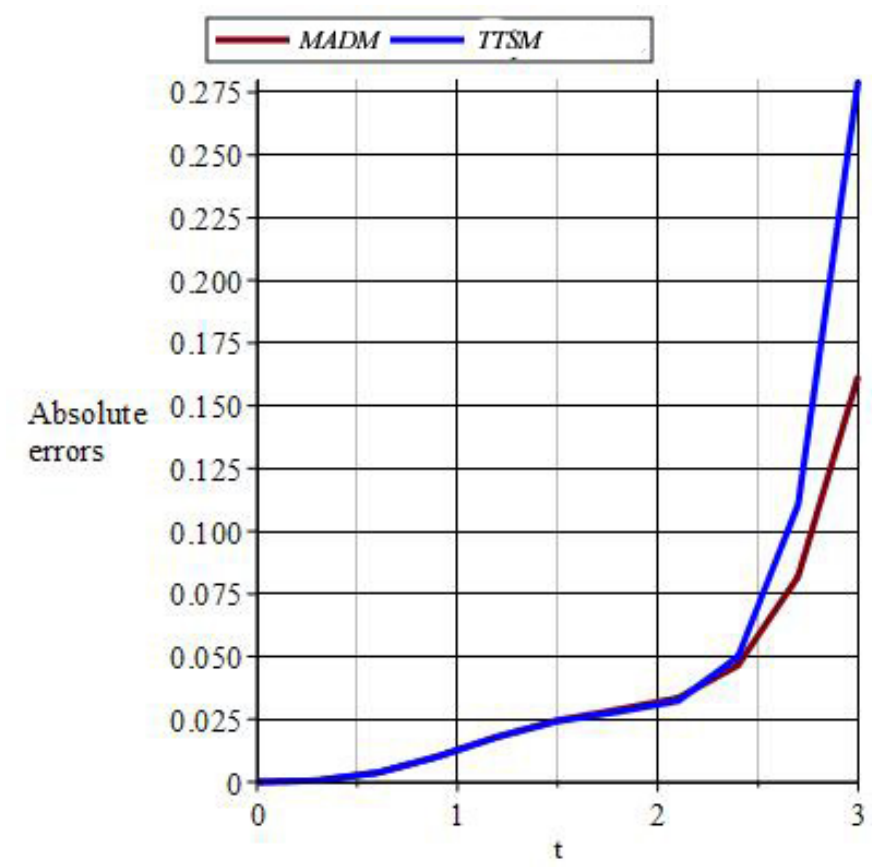

Figure 5: Plot of absolute errors from approximating polynomials (3) and (12) of TTSM and 4-component MADM.

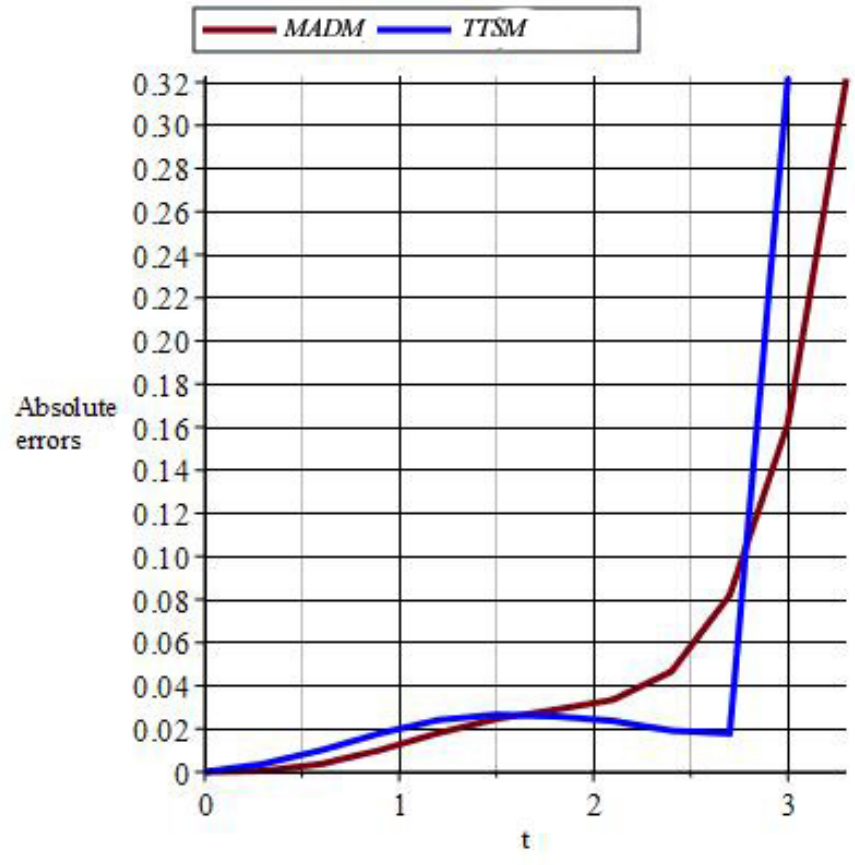

Figure 6: Plot of absolute errors from approximating polynomials (4) and (12) of TTSM and 4-component MADM.

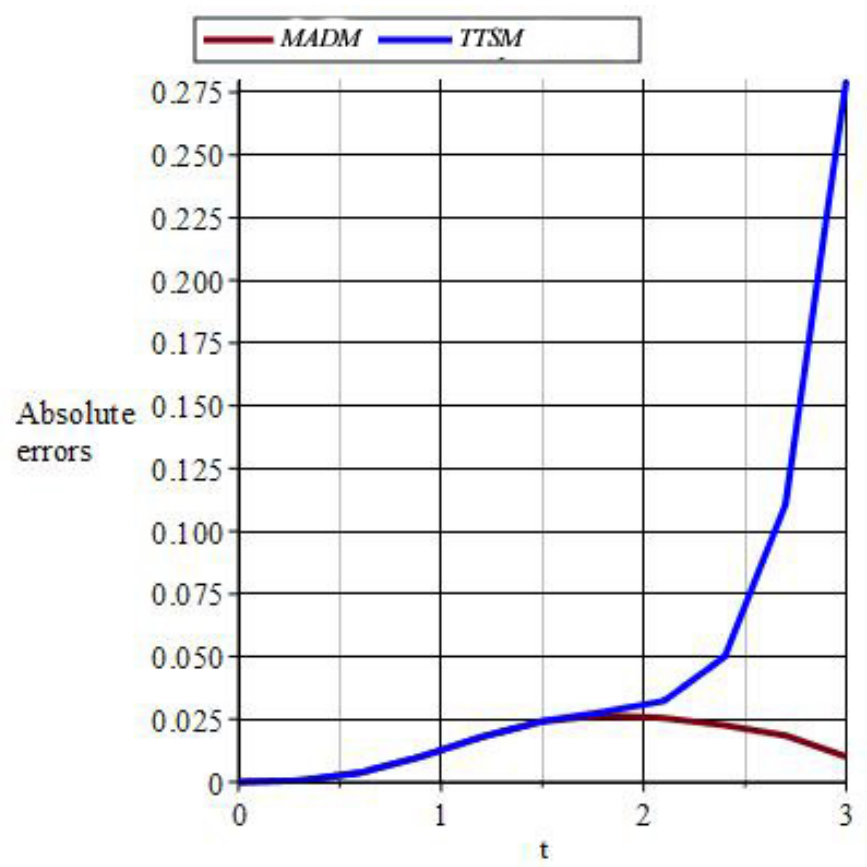

Figure 7: Plot of absolute errors from approximating polynomials (3) and (14) of TTSM and 5-component MADM. 


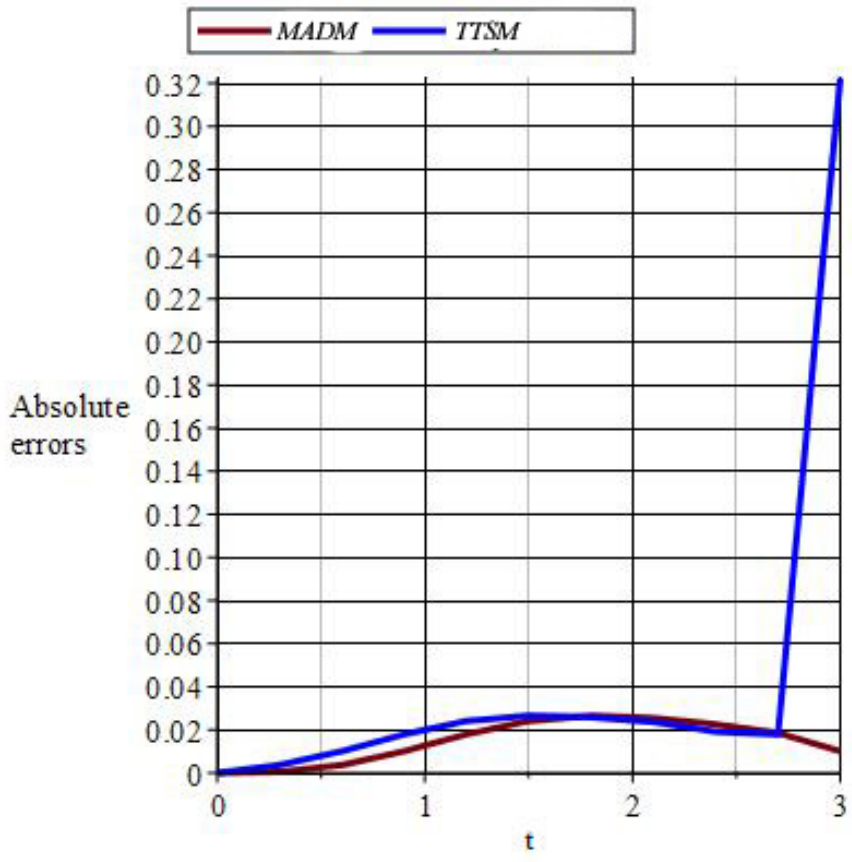

Figure 8: Plot of absolute errors from approximating polynomials (4) and (14) of TTSM and 5-component MADM.

Equation (12) of approximating 4-component MADM behave more like approximate P-L method than approximating polynomial (3) of TTSM as shown in Figure 1 and Table 1. On the other hand, many more terms are added to approximating TTSM (3) to obtain TTSM (4). Solutions from approximating polynomial (4) of TTSM with twenty-two order behave more closely as that of approximate P-L method than equation (12) of approximating 4-component MADM series and are indicated in Figure 2 and Table 2. However, results from 5-component MADM (14) shown in Tables 3 and 4 are similar to the results obtained from approximate P-L method than those got from TTSM of equations (3) and (4) as shown in Figures 3 and 4. The absolute errors generated by the approximating polynomial (3) are greater than the ones generated by (12) as shown in Figure 5. Absolute errors obtained from TTSM (4) are generally lower than the ones generated from (12) of MADM due to many numbers of terms added to TTSM (3) as shown in Figure 6. Finally, Figures 7 and 8 indicate that (14) shows smaller absolute errors compared with (3) and (4). At $\mathrm{t}=3$ from Table 1, equation (3) gives $u(3) \approx-1.27$ and (12) gives $u(3) \approx-1.15$. Observe that with several terms added to (3) to obtain (4), the solution obtained in Table 4 at $t=3$ for (4) is not as close to that of approximate P-L method as (14) which is obtained from (12) by addition of just one component. The theoretical implication of this is that few components from MADM - obtained with ease - can produce solution that is more convergent to that of $\mathrm{P}$ $\mathrm{L}$ method than for tediuos computational work of many terms added to TTSM.

\section{Conclusion}

In this paper, approximate series solutions of the van der Pol equation have been obtained using the truncated Taylor series method and the modified Adomian decomposition method. The two series solutions for Van der Pol equation obtained as approximating polynomials (3), (4), (12) and (14) agree. From the approximating polynomials of the two series solutions, MADM produces more convergent results to the P-L methods than the TTSM, as only very few components of the MADM yielded a series much longer than order twenty of Taylor series. Hence, we recommend inclusion of the MADM in modern mathematical tools.

\section{References}

[1] E. A. Az-Zobi, K. Al-Khaled \& A. Darweesh, "Numeric-Analytic solutions for nonlinear oscillators via the modified multi-stage decomposition method.", MDPI Journal of Mathematics 7 (2019) 1. doi: 10.3390/math7060550

[2] J. Biazar and Y. Shafiof, "A simple algorithm for calculating Adomian polynomials. Int. J. Contemp. Math. Sciences, 975 - 982.

[3] Y. Daoud \& A. A. Khidir, "Modified Adomian decomposition method for solving the problem of boundary layer convective heat transfer. Journal of Propulsion and Power Research 7 (2018) 231. doi: 10.1016/j.jppr.2018.05.005

[4] I. L. El-Kalla, "Error analysis of Adomian series solution to a class of nonlinear differential equations", Applied Mathematics E-notes 7 (2007) 214.

[5] H. Gundogdu \& O. F. Gozukizil, "Solving nonlinear partial differential equations by using Adomian decomposition method, modified decomposition method and Laplace decomposition method", MANAS Journal of Engineering 5 (2017) 1.

[6] J. H. He, "Modified Lindstedt-Poincare methods for some strongly non-linear oscillations. Part 1: expansion of a constant", International Journal of nonliear mechanics 37 (2002) 309. doi: 10.1016/S00207462(00)00116-5

[7] K. G. Howison, Practical Applied Mathematics: Modelling, Analysis, Approximation", (2005) Cambridge University Press, United Kingdom.

[8] B. Jang, "Two-point boundary value problems by the extended Adomian decomposition method", J. Comput. Appl. Math 219 (2008) 253. doi: 10.1016/j.cam.2007.07.036

[9] K. K. Kataria \& P. Vellisamy, "Simple parameterization methods for generating Adomian polynomials", Applicable analysis and discrete Mathematics 10 (2016) 168. doi:10.2298/AADM160123001K

[10] J. D. Logan, Applied Mathematics., (2006) John Wiley.

[11] P. J. Melvin, "On the construction of Poincare-Lindstedt solutions: The nonlinear oscillator equation", SIAM Journal of Applied Mathematics 33 (1977) 161. doi:10.1137/0133011

[12] D E. Panayotounakos, N. D. Panayotounakou \& P. S. Vakakis, "On the lack of analytic solutions of the van der Pol Oscillator, ZAMM - Journal of Applied Mathematics and Mechanics 83 (2003) 611. doi: 10.1002/zamm.200310040

[13] Y. W. Putranto \& S. Mungkasi, "Adomian decomposition method for solving population dynamics model of two species", Journal of Physics: Conference Series 795 (2017) 1. doi: 10.1088/1742-6596/795/1/012045

[14] U. Saeed, "Haar Adomian method for the solution of Fractional Nonlinear Lane-Emden type equations arising in astrophysics", Taiwanese Journal of Mathematics 21 (2017) 1175. doi:10.11650/tjm/7969

[15] A. S. Soomro, G. A. Tularam \& M. M. Shaikh, "A comparison of Numerical Methods for Solving the Unforced Van Der Pol's Equation", Mathematical Theory and Modelling 3 (2017).

[16] D. Viswannath, "The construction of Lindstedt-Poincare technique as an algorithm for computing periodic orbits, SIAM Journal of Applied Mathematics 43 (2001) 478. doi: 10.1137/s0036144500375292

[17] A. M. Wazwaz, "A comparison between Adomian decomposition method and Taylor series method in the series solutions", Appl. Math. Comput 97 (1998) 37. doi: 10.1016/S0096-3003(97)10127-8 
[18] A. M. Wazwaz "A new algoritm for calculating Adomian polynomials for nonlinear operators", Appl. Math. Comput 111 (2000) 53. doi: 10.1016/S0096-3003(99)00063-6

[19] A. M. Wazwaz \& A. Gorguis, "An analytic study of Fisher's equation by using Adomian decomposition method", Appl. Math. Comput 154 (2004) 609. doi: 10.1016/S0096-3003(03)00738-0 